\title{
CyberBridges A Model Collaboration Infrastructure for e-Science
}

\author{
Heidi L. Alvarez, David Chatfield, Donald A. Cox, Eric Crumpler, Cassian D'Cunha, Ronald Gutierrez, \\ Julio Ibarra, Eric Johnson, Kuldeep Kumar, Tom Milledge, Giri Narasimhan, S. Masoud Sadjadi, Chi Zhang \\ Center for Internet Augmented Research and Assessment (CIARA) \\ Florida International University, Miami, FL 33199
}

\begin{abstract}
The "CyberBridges" pilot project is an innovative model for creating a new generation of scientists and engineers who are capable of fully integrating cyberinfrastructure into the whole educational, professional, and creative process of their respective disciplines. CyberBridges augments graduate student education to include a foundation of understanding in Advanced Networking and Grid Infrastructure for High Performance Computing, and bridges the divide between the information technology community and diverse science and engineering disciplines. We demonstrate the effectiveness of CyberBridges by providing four case studies. Groundwork has begun to extend the outreach of CyberBridges for international research and education collaborations.
\end{abstract}

\section{Introduction}

A significant challenge exists in expanding the impact of cyberinfrastructure (CI), i.e. infrastructure of Grid and high-speed networks. We conducted a pilot project within the Center for Internet Augmented Research and Assessment (CIARA) at Florida International University (FIU). CyberBridges mission is to create a new generation of scientists and engineers who are capable of fully integrating CI into the whole educational, professional, and creative process of diverse disciplines.

The concept of e-Science [32] has been proposed to develop and sustain global networks of scientists conducting collaborative research. The ambition of eScience is to help increase the rate of discovery by empowering scientists with CI that will help them leverage resources on a global scale. The underlying premise is that the endeavor of science and discovery is too large for individual scientists and laboratories. In order to address fundamental research issues in phenomenon such as Astronomy, Bio-Diversity, Epidemiology, and High-Energy Physics collaboration is necessary. Moreover, the "collision of ideas", envisaged by Bruce Alberts, and the resulting creative tension inherent in diversity, needs to be harnessed to increase the rate of innovation and discovery [3].

From a US perspective, traditionally, colleges of science and engineering in the US have relied on international graduate students, from countries such as China, India, and Brazil, to provide a pool of research labor and potential recruits for future academic and industrial R\&D positions. However, with the recent decline in applications from qualified candidates from these traditional countries, it becomes imperative that investigators in US universities reach out and collaborate with researchers and graduate students in their home countries. This further underscores the need for underlying $\mathrm{CI}$ that can help national researchers cooperate seamlessly with their counterparts in other countries [29].

However, the normative state is that CI research scientists (institutional information technology professionals with academic backgrounds) have worked hard to ensure that $\mathrm{CI}$ is in place to maintain low barriers for use of these technologies, but lack topical expertise to explain how CI may be of benefit to other disciplines. Early adopter faculty members are excited to see potential benefits in their research domains, but they are the exception. The majority of faculty members do not see the applicability of CI for their own domains. Scanning other domains for possible benefit and collaborations is often a desired activity, but too much of a time burden and often the technical material is difficult to apply [14]. Previous efforts have tried to work with these early adopters to become promoters for $\mathrm{CI}$ in entire domains of science. That work did not have a long or broad impact, as it depended on enthusiasm without a system for substantive rejuvenation $[5,28]$.

The current initiatives in developing $\mathrm{CI}$ have focused primarily on building technologies such as computational grids connected to multi-gigabit optical networks, or access grids that allow remote conferencing. For successful adoption and use of these 
technologies, these efforts need to be complemented with the development of appropriate coordination mechanisms and socio-organization protocols and their dissemination to the scientific community through tailored educational programs and training.

The CyberBridges project is a model collaboration infrastructure for e-Science, designed to complement the technical infrastructure with a socio-organizational service-oriented infrastructure. It offers the potential of creating a community of scientists and researchers capable of collaborating with their counterparts across the network by fully integrating CI into their educational, professional, and creative processes. The "collisions of ideas", which Bruce Alberts identified as requisite to faculty and student success, is the core tenant of CyberBridges [3]. Thus, the project includes the development, deployment, and testing of the technical infrastructure as well as the coordination, workflow, and social protocols needed for crosscultural/cross-national collaboration.

To summarize, CyberBridges has three objectives:

(a) To develop and test a model socio-technical cyberinfrastructure for enabling and sustaining research collaborations among networks of distributed science and engineering researchers.

(b) To develop a prototype community of distributed science and engineering researchers.

(c) To integrate the researchers, graduate students and faculty from the minority communities in the United States with the research community.

In this paper, we demonstrate the effectiveness of CyberBridges by providing four case studies with graduate students of Physics, Bioinformatics, Chemistry, and Biomedical Engineering. The rest of this paper is organized as follows: In Section 2, we present the existing works at FIU. Our approaches and methodologies are outlined in Section 3. The project implementation, case studies, and educational assessments are given in Sections 4, 5, and 6, respectively. Section 7 concludes the paper and Section 8 discusses the future work.

\section{Existing work at FIU}

\subsection{AMPATH}

CIARA $^{1}$ houses the AMPATH ${ }^{2}$ research and education networking international exchange point in Miami, serving South and Central America, the Caribbean and Mexico, as well as a variety of US research programs in the region. AMPATH is aided by the presence of one of only five Tier-1 Network

\footnotetext{
1 www.ciara.fiu.edu

2 www.ampath.net
}

Access Points (NAP) in the US. In addition, CIARA leverages FIU's networking expertise and its membership in the Open Science Grid (OSG) through its AMPATH research network activities.

\subsection{PAC}

Recent national reports have indicated a continuing major problem of student drop out which particularly impacts underrepresented, minorities [30, 10, 25]. Our research at FIU during the past nine years with grades 7-12 "at-risk" students of an urban public school district clearly indicates the strong potential that instructional technology used in mathematics and science contexts has been improving the academic success rates of this population.

\subsection{CHEPREO}

FIU, together with partners at Florida State University, the University of Florida, the California Institute of Technology, international partners in Brazil, in cooperation with the National Science Foundation, operates an interregional Grid-enabled Center for High-Energy Physics Research and Educational Outreach $\left(\right.$ CHEPREO $\left.^{3}\right)$ at FIU. The CHEPREO team continues to make outstanding contributions in the development of the Compact Muon Solenoid experiment at CERN while equally engaged in educational outreach efforts at the high school, undergraduate and graduate level.

\subsection{WHREN-LILA}

The NSF Office of Cyberinfrastructure International Research Network Connections program funds the Western Hemisphere Research and Education Network $(\mathrm{WHREN})^{4}$ Links Interconnecting Latin America (LILA) which addresses the existing and future needs for improved North American (especially U.S.)-South American connectivity and specifically focuses on the need for connectivity through new links. The WHREN-LILA team has brought international domain science and CI breadth of knowledge as well as their collaborations to bear on the CyberBridges model.

\section{Methodologies}

\subsection{Approaches to e-Learning Sciences}

CyberBridges uses "modeling" in instruction and learning. Mathematical modeling is a form of

\footnotetext{
${ }^{3}$ www.chepreo.org

${ }^{4}$ www.whren-lila.net
} 
mathematical problem solving that produces solutions to complex and real, applied problems by creating theoretical representations of the real situation. It is frequently a systems approach to solve a problem that requires the confluence of varied linked strategies. The inquiries that the CyberBridges fellows construct with their faculty advisors model these connections through computer simulations. Technology permits teams of individuals, possibly in different locations and with different skills to the work toward solution of the real problem. The NCTM "Mathematics as Problem Solving" Standard conceptualizes mathematical modeling (see Figure 1).

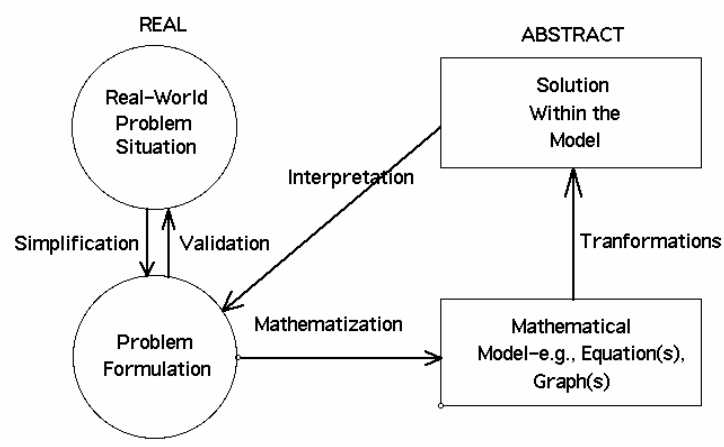

Figure 1: Mathematical modeling

Research clearly indicates the high potential of the modeling approach for improving learning. Students learn best when the dynamic and developmental process of learning is guided by generalized principles applied to real-world problems or situations [4]. Key themes relevant to teaching and learning found in cognitive psychology, including the idea that learning is a constructive process and that meaningful learning is contextual [21], indicates that activities must encourage students to become personally and actively involved in their own learning. The modeling approach provides just such an atmosphere in which students solve problems, make decisions, work with their peers, and pursue new learning as ideas evolve. We believe that graduate students constructing the models with faculty advisors and IT research scientists has provided the context, and the exploration of the inquiry is engaged personal learning.

\subsection{Project structure}

CIARA was responsible for the outreach programs (department meeting presentations, brown bag lunches, and conferences), soliciting fellowship proposals, administering the graduate certification program, and providing the IT tools and expertise for the structured inquiry projects.
The project offered four, two semester long, fellowships. Each fellowship started with a normative analysis, then an inquiry project, followed with a case study package. In addition, each fellow was required to successfully complete the certification program, which includes two courses taught by professors and research scientists from FIU School of Computing and Information Sciences. The inquiry-based project does not have to meet any prerequisite of success, rather thoroughness is required. The project was structured so that each graduate fellow has a faculty advisor (typically their thesis advisor in his/her major and one research scientist advisor of CI.)

There was one advisory committee of solicitation review, and one external assessment and reporting committee who were engaged to annually review the progress of CyberBridges and further ensure academic integrity. The CyberBridges advisory committee consisted of three sciences, engineering and sponsored research members. Both committees insured that the IT research proposed by CIARA research scientists was applicable and current. The operational milestones of CyberBridges come from the execution of the fellowship activities, and the annual assessment of the delta from the past normative states.

\subsection{Hypothesis and assessment}

The CIARA CyberBridges proposed that graduate students engaged in inquiry-based learning activities can affect transfer of CI research and that this transfer will increase scientists' rates of discovery and create a CI empowered workforce.

Measuring CyberBridges' project successes falls under a number of rubrics. When a project is begun with a faculty member, a graduate student, and a CI research scientist, the first course of action is to document the normative state. As the metrics for progress vary significantly by domain, this process is a highly customized activity. Different faculty members may choose to judge their progress in different ways even inside of domains. CyberBridges documents this, and then uses it as a baseline. The success of CyberBridges implementation is then an assessment of the defined metrics.

CyberBridges calls for an external assessment committee comprised of educational assessment and subject matter experts to perform a Baseline Study when the fellows are selected. The external assessment committee was contracted to measure the pedagogy against known best practices, and the technology against the current work of the CI research community, as well as auditing the operations of the CyberBridges implementation. 


\section{Project implementation}

\subsection{Continuous cross-disciplinary dialogue}

The investigators developed relationships with the CI research community, and were active in working groups that seek to form applications of CI research. CIARA CI research scientists worked with faculty in various disciplines to find matches between research interests and CIARA projects. These collaborations assisted CI researchers in understanding needs, and challenges in applying their work.

CIARA CyberBridges presentations to faculty have fostered an environment of continuous dialogue. The FIU faculty communities have been engaged creatively to understand applications of CI research. This environment is a fertile space for multi-disciplinary research to be initiated. The open exchange will be a model for institutions that replicate CIARA's CyberBridges.

\subsection{Collaborative proposal writing}

CyberBridges projects came from a one-page proposal document, collaboratively authored by a $\mathrm{PhD}$ fellowship candidate and their faculty advisor in their respective domains. The CI research scientists engaged faculty and students by offering an introductory workshop providing, details on how to apply for a fellowship. The fellowship proposal described the nature of the domain specific problem and a particular inquiry into the problem for which a graduate student can explore applicability of CI. These proposals were not meant to be prescriptive. The use of CI in the inquiry was hypothetical. The graduate student researched and experimented to determine the veracity of the hypothesis. The one page document was meant to be a low enough barrier that many faculty members could explore fellowships early in their exploration of topics. These one-page proposals were also used as a metric for CyberBridges implementation success. The fellowships are two semesters long plus travel to an international conference to present results.

\subsection{Faculty review of proposals}

The one-page proposals were brought forward to a faculty advisory committee. The committee evaluated the proposals based on the prospects for CI augmentation, the chance of being replicated and the scholastic suitability of the proposed inquiry. The faculty committees ensured that the nature of the proposed research can hold up to peer review. The committee then ranked the proposals and offered written critiques.

\subsection{Normative assessment}

CIARA CI research scientists, with the graduate fellow and the faculty member, documented discipline specific metrics of success. Often these are the same metrics used in tenure and promotion. There are domain specific variants that are taken into account. This normative document is used at the conclusion of the fellowship period to explore potential changes. CyberBridges publishes detailed assessment activities on the web site.

\subsection{Nesting of graduate students in lab}

The graduate students worked with the CI research scientists to explore the challenge put forward in the one page proposal. This exploration has taken place in a lab environment, where there were graduate students of various disciplines, all exploring applications of CI research into their domains. This multi-disciplinary environment is a fertile space for cross fertilization of approaches and understandings. Under the tutelage of the CI research scientists, the graduate fellows have been engaged in a rich inquiry-based learning experience.

\subsection{CI scholastic certification program}

CIARA's CyberBridges fellowship program has three requirements. One is the inquiry-based project that involves the faculty sponsor. The second is a course of study leading to a certificate in scientific networking and computing. The certification program requires two 3-credit courses. Based on the existing Grid Computing lectures developed in the School of Computing and Information Sciences at FIU, we have developed a new graduate-level Grid Computing course. The course includes lectures, seminars, and a course project. The lectures cover topics such as HighSpeed Networks, Design and Implementation of Clusters, Globus Toolkit, Virtual Data Systems, Resource Scheduling, MPICH-G2, Grid Services, and Job Workflow Processes. The course project requires students to "gridify" a scientific application. Students are required to conduct experiments on the CyberBridges cluster, and leverage extramural resources, such as TeraGrid and Open Science Grid, as applications might justify. The courses will be added into the graduate course catalog thereby institutionalizing CyberBridges at FIU.

After the basic Grid Computing course, the students took an independent-study course to conduct interdisciplinary research. The course provided a solid foundation of understanding about how CI research 
takes place, and how to be involved in the applications of it. While many faculty members today are not aware of the power of CI, the graduate fellows in their careers as faculty members will have a rich understanding of the CI community. Permanent Equipment for the certification program includes both high performance networking and grid computing training components. Figure 2 provides details as to what is available and how it is set up.

This certification program is an important tool for institutions apart from FIU to directly benefit from CIARA's CyberBridges initiative. As the graduate fellows interview for post-doctoral positions, outside programs will be able to determine if such expertise could be beneficial for their departments. This exponentially increases the scope of impact of CIARA. The combination of an inquiry-based learning program and the certification program will best prepare the next generation of scientists, engineers and educators to use and understand IT research.

\subsection{Case study package}

The graduate fellows have worked with CIARA CyberBridges research scientists and the faculty advisors to author a case study package. This package contains all of the assessment data, the domain specific challenge and history of the inquiry, and the technologies employed. The case study package is detailed so that others can use it in the domain of the graduate fellow and faculty member to replicate the inquiry. The case study package represents examples across the disciplines for applications of CI research.

"Best Practices" white papers are useful in conveying to large communities how to employ applications of CI research in video conferencing [12]. The case studies can be a reference that is technically accessible to specific domains. An inquiry, based in high-energy physics, for example, has resulted in a case study package that is easily understood by other physicists. The case studies will themselves be mechanisms to effectively transfer CI research into the domains in a widely available and applicable form.

\subsection{Student outreach}

The graduate fellows are required to present their work with CI research to their peers. The graduate fellows will work with their peers to present the role of CI research. Having graduate students that are knowledgeable about a topic and acting as role models for undergraduate students has proven to be effective in minority serving institutions [30]. In disciplines that have not effectively appealed to minorities, we believe that CI research may offer a new perspective as to the excitement in those fields. By having role models convey their enthusiasm in using CI research in the humanities or physical sciences, the opportunity for impact is significant [30].

\section{Case Studies}

We demonstrate the effectiveness of CyberBridges by providing four case studies with graduate students of Physics, Bioinformatics, Chemistry, and Biomedical Engineering. Each graduate student worked with CIARA CyberBridges research scientists and the respective faculty advisors to author a case study package. Under the supervision of the CIARA research scientists, the students built a high-end CyberBridges cluster, and conducted e-science experiments of High-Performance Grid Computing (see Figure 2). The four on-going projects are described as follows.

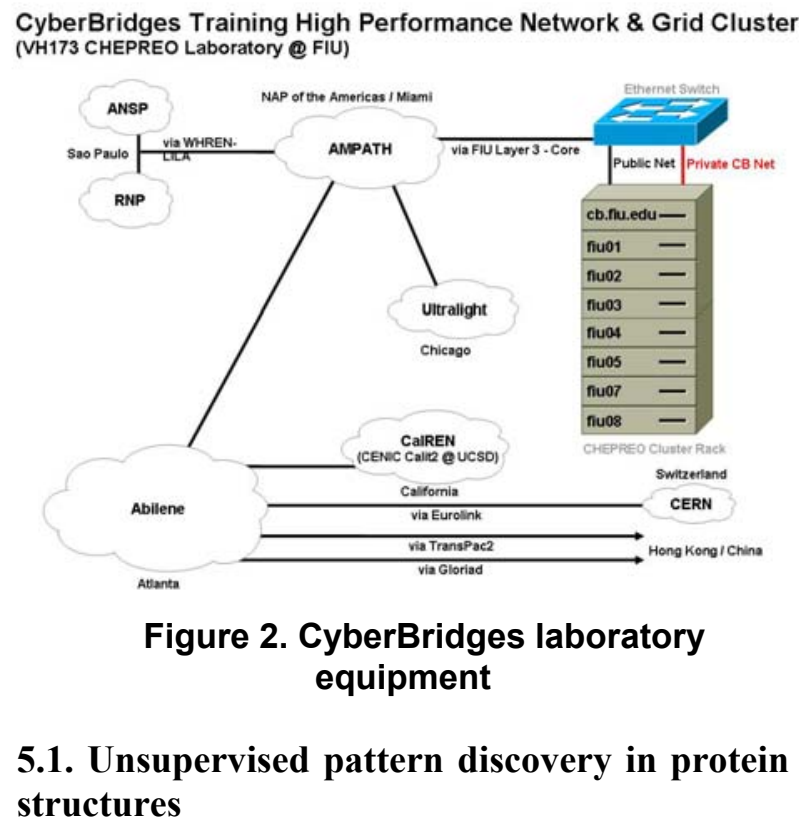

This is a partnership among Dr. Giri Narasimhan, Computer Science \& Bioinformatics, graduate student Tom Milledge, and the CyberBridges team. Pattern Discovery refers to the task of identifying relevant and significant commonalities or essential differences in related data. In bioinformatics applications, patterns come in different flavors. in sequences, structures, shapes, images, and in quantitative and temporal data. Since nature tends to reuse these patterns, pattern discovery is an important task in understanding the nature around us. The aim is to implement unsupervised pattern discovery tools for protein structure data. There are two computational tasks. First, a database of proteins and sequence information is searched to look for protein structures that appear to share potentially useful functions. 3-D visualization 
best reveals these functions. Then the results are placed in an output "metadatabase" (with geometric hashing allowing fast search/retrieval of 3-D structure). Second, the output database will be searched to locate useful structures. The output database is very large, but it has been indexed to enable efficient searches. An effective job done by the algorithms in the first task will produce a relatively smaller and manageable output database.

A prototype version of the application was written in a mixture of $\mathrm{C}$ and $\mathrm{C}++$ code. Most of management code was written in $\mathrm{C}++$, with Matrix utility routines implemented in $\mathrm{C}$ for performance reasons. For the search component, Milledge has conducted a number of experiments using MPI-based message passing search strategies, as well as another method using the global shared memory GA Toolkit (http://www.emsl.pnl.gov/ docs/global/). He has used the implementation of Global Arrays on the Blue Gene development machines in IBM Rochester, where he was an intern over the summer 2006.

\subsection{Modeling biological tissue scaffolds in three dimensions}

This is a partnership among Dr. Eric Crumpler, Biomedical Engineering, graduate student Ronald Gutierrez, and the CyberBridges team. Bioreactors are used as cell culture systems for temporary growth and maintenance of tissue-engineered constructs that serve as three-dimensional templates for initial cell attachment and subsequent tissue formation. The bioreactors' dynamic environment is known to play a crucial role on the cellular components synthesis like glycosaminoglycans (GAG). Systematic research of the Wall Shear Stress (WSS) effects on the scaffold's shape has been limited. Results of this research will allow for a detailed understanding of the effect of hydrodynamic forces and nutrient mass transfer on the surface of scaffolds (as well as within) that affect the cell growth and further tissue formation. Hence, cyberinfrastructure advantages are invaluable in the applicability of the new and composite simulations: first, to enrich the knowledge on bioreactor complex fluid flow; and second, to design more efficient and hydrodynamic scaffolds for enhanced tissue growth understanding. The objective is to develop computational models for scaffolds' geometries and compare WSS values to evaluate performance using the results as aid for design selection.

\subsection{Interplay between random matrix theory and quantum field}

This is a partnership among Dr. Rajamani S. Narayanan, Physics, graduate student_Alejandro de la Puente, and the CyberBridges team. The goal for physicists is to determine the fundamental parameters that describe each particle in the current standard model and the physics required exceed traditional Quantum Field Theory. Quantum Chromodynamics (QCD) is a theory that has proven to be very accurate in the determination of these parameters. Lattice Quantum Chromodynamics (LQCD), which is a discretized version of QCD, replaces the continuum with a four-dimensional grid for its calculations. LQCD is a challenging computational field that employs large scale numerical calculations, and will be used to verify the current standard model. The aim of this project is to develop the required computational capability and precision using random matrix theory. This is a more complete, accurate (as well as more computationally intensive) approach to identify particles in data that will be generated by the CERN Large Hadron Collider (LHC) experiments beginning in 2007. The task is to organize and divide up the matrices/computational tasks so that Grid computing can be applied. Results will be compared to the currently well accepted LQCD simulation.

\subsection{Functionalities of a specific enzyme for certain reactions}

This is a partnership among Dr. David Chatfield, Chemistry/Biochemistry, graduate student Cassian D'Cunha and the CyberBridges team. Computer simulations of biological macromolecules are widely used in the pharmaceutical industry to accelerate drug discovery and in academic research for a host of problems from protein folding to enzyme mechanism. The sizes of the molecular systems and the time scales modeled are memory and computation intensive. Computer clusters have become popular for such applications, either for running many simultaneous simulations to obtain good sampling statistics or for distributing a single, expensive calculation over many computers. This research is focused on an enzyme, chloroperoxidase (CPO) [31], which is of industrial interest, as a potential biological chiral catalyst. It is aimed at elucidating the reaction mechanism of CPO and at predicting useful site-specific mutations for fine-tuning the enzyme's catalytic activity for particular substrates of interest. The calculations that we need to perform are of two types, both of which require substantial computer power: quantum mechanical (QM) calculations for the mechanism work, and molecular mechanical (MM) calculations for the mutation work.

D'Cunha has set up a Linux cluster and implemented clustering operating system software on 
the basis of instruction received in the CyberBridges program. QM and MM software (CHARMM, Turbomole and Gaussian) have been installed. The parallel implementation of CHARMM has been benchmarked with a macromolecular simulation. Currently the parallel implementation of Turbomole and Gaussian is under investigation.

\section{Educational assessment}

The progress reports indicate that the students are all making significant headway toward having their research computation fully implemented on a cluster. In each case the application of cluster computing is a significant change in their research procedures, and takes considerable learning, programming, benchmarking/testing. The research problems tackled and approaches used are state-of-the-art in their respective fields. As the projects continue, normative metrics of research publications and presentation will be applied. A next step for the evaluators is to look at the publication norms for each of the teams' fields and determine what level of publication would be evidence of the value added to their research by participation in the CyberBridges program.

The reports on each of the specific research projects give evidence for the success of the two classes that taught the graduate students the computer science background to understand cluster computing and the hands-on work of building a cluster. In each case, they were able to participate in building a cluster in class, and two of the teams have versions of their models working on them. All teams are focused on the effort of parallelizing and programming their models to run on clusters of a size that will result in significant improvements in their research productivity.

Furthermore, the success of the classes with the four teams here indicates the potential value of linking a more global application of CyberBridges with much of what is happening in the classes operating more widely through e-collaboration and virtual cluster computing labs. Another potential for e-collaboration is that graduate students often pave the way for increased faculty e-collaboration.

The preliminary conclusion for this evaluation is participation in the CyberBridges program has been advantageous for the research of each team, and the program structure and classes worked well. In each case, there is an evident prospect for more rapid and computationally advanced research production. Significant benefits could be obtained by an expanded version of the program, particularly if it incorporates the e-science/e-collaboration facilities now available at FIU.

\section{Conclusions}

The smallest impact of CIARA's CyberBridges is a change in the way that FIU, the largest Hispanic minority-serving institution in the continental U.S., views cyberinfrastructure. Where CI has raised the tide, not all vessels have been lifted. CIARA brings the benefits to domains and groups that have yet to realize the fantastic impact. This shift will result in scientists, engineers, and educators who are fully integrating CI into their professions.

\section{Future Work: Global CyberBridges}

The CIARA model will likely have a much larger impact: a Global CyberBridges project (GCB). The adoption and use of $\mathrm{CI}$ is further complicated by the fact that collaborations between globally distributed researchers are subject to a variety of global "Gaps" between the work-sites: distance and its associated attributes of culture, time zones, geography, language, and social protocol affect how humans interact with each other $[8,26]$. Global CyberBridges will help in two ways - it will develop a body of experience, guidelines, and theory that will be useful in designing global research communities in the future. Second, it will also help build such collaboration between scientists from the US, China, and Brazil, nations with very different cultures, traditions, and infrastructures. Furthermore, we will be developing a community - as a proof of concept - and while building this community we will also create a body of knowledge that will be useful for building future e-Science communities.

Acknowledgements CyberBridges was supported by NSF award OCI-0537464. Global CyberBridges has been funded for three years (October 2006 - December 2009) by NSF award OCI-0636031.

\section{References}

[1] Atkins et al., Revolutionizing science and Engineering through Cyberinfrastructure: report of the National Science Foundation Blue-Ribbon Panel on Cyberinfrastructure. Arlington, Va. The National Science Foundation, 2003.

[2] Bransford, John, Ann L. Brown, and Rodney Cocking (Eds.). How People Learn: Brain, Mind, Experience, and School. Washington DC: National Academy Press, 1999.

[3] Boyer Commission on Educating Undergraduates in the Research University. Reinventing undergraduate education: A blueprint for America's research universities. Menlo Park, CA: Carnegie Foundation for the Advancement of Teaching, 1998. 
[4] Bruning, R., Schraw, G., \& Running, R, Cognitive Psychology and Instruction. New York, New York. Prentice Hall, 1998.

[5] Cambre M.A., The state of the art of instructional television. (pp. 267-275), In G.J. Anglin (ed) Instructional Technology, Past, Present and Future. Englewood Cliffs: Prentice-Hall, 1991.

[6] COSMOlogic GmbH \& Co. HG. Turbomole 5.6. Burscheider Str. 515 D-51381, Leverkusen. Germany.

[7] Dumas, Marlon, Wil M. P. van der Aalst, and Arthur H.M. ter Hofstede, Process-Aware Information Systems: Bridging People and Software through Process Technology, Wiley-Interscience, p. 14, 2005.

[8] Engestrom, Y., Engestrom, R., \& Karkkainen, M, Polycontextuality and boundary crossing in expert cognition: Learning and problem solving in complex work activities. (Vol. 5), 1995.

[9] Foster, Ian. Viewpoint: Service-Oriented Science, Science: Vol. 308. no. 5723, pp. 814-817; DOI: 10.1126/science.1110411, May 2005: http://www.sciencemag.org/cgi/content/abstract/308/5723/81 4

[10] Frase, M., Kaufman, P., \& Klein, S., Dropout rates in the United States: 1997. Washington, DC: National Center for Education Statistics. NCES 1999-082, April, 1999.

[11] Geles, C., Lindecker, G., Month, M., \& Roche, C., Managing Science; Management for R\&D Laboratories. A Wiley-Interscience Publication. John Wiley \& Sons, Inc, 2000 .

[12] Gemmil \& etc., The Videoconferencing CookBook. Birminghan, Alabama, 2003.

[13] Guba, E. G. \& Lincoln, Y., Fourth generation evaluation. Newbury Park, California. London, England. New Delhi, India: Sage Publications, 1989.

[14] Hazemi, R., Hailes, S., \& Wilbur, S., The Digital University: Reinventing the Academy. Berlin: SpringerVerlag, 1998.

[15] Joy, Bill, Kennedy, Ken \& etc., The Presidents Information technology Advisory Board: Report to the President. Washington, D.C. National Coordination Office, 1999.

[16] Kaufman, P., Klein, S., \& Frase, M., Dropout Rates in the United States, 1997. Statistical Analysis Report. U.S. Department of Education, 1999.

[17] Kumar, K., and P.C. van Fenema, Passing the Baton cross the Divide: Distance, Interdependence, and Coordination in Global Software Development. FSO (Financial Services Outsourcing) Magazine, http://www.fsoutsourcing.com 1 (2), 2004.
[18] Lamdan, Y., Wolfson, H.J. Geometric hashing: A general and efficient model-based recognition scheme, International Conference on Computer Vision, 1988, 238249.

[19] Lee, Hong-In, Dexter, Annette F., Fann, Yang -Cheng, Lakner, Frederick J., Hager, Lowell P., \& Brian M. Hoffman. J. Am. Chem. Soc. 1997, 119, 4059-4069

[20] Leibowitz, N., et al., Automated multiple structure alignment and detection of a common substructural motif. Proteins, 43, 235 V245, 2001.

[21] Mcombs, B. \& Whisler, J., The Learner-Centered Classroom and School: Strategies for increasing Student Motivation and Achievement. Jossey-Bass, 1998.

[22] Mumford, E. "Socio-Technical Design: An Unfulfilled Promise or a Future Opportunity," in R. Baskerville, J. Stage, and J.I. DeGross (Eds.), Organizational and Social Perspectives on Information Technology, Boston: Kluwer Academic Publishers, 2000, pp. 33-46.

[23] NAEP 1996 Mathematics Report Card for the Nation and the States: Findings from the National Assessment of Educational Progress. Washington, DC: National Center for Education Statistics.

[24] National Council of Teachers of Mathematics (NCTM), Curriculum and evaluation standards for school mathematics. Reston, Va.: NCTM, 1989.

[25] Natriello, G., McDill, E. L., \& Pallas, A. M., Schooling disadvantaged children: Racing against catastrophe. New York: Teachers College Press, 1990.

[26] Olson, G. M., \& Olson, J. S., Distance matters, science of collaboratories. Human Computer Interaction, 15, 139179, 2001. Retrieved from http://www.scienceofcollaboratories.org/WorkshopStuff/June 2001/pdfs/Olson_Olson_distance_matter.pdf.

[27] Olson, G. M., Malone, T. W., \& Smith, J. B., Coordination Theory and Collaborative Technology, Lawrence Erlbaum Associates, 2001.

[28] Pournelle J., An Educational Trip. Byte, 197-210, 1994.

[29] Negraponte, N., Being digital, Random House, New York, 1995.

[30] Stearns, C. \& Snyder, T, Hispanic Serving Institutions Statistical Trends form 1990 to 1999. National Center for Educational Statistics. Washington, D.C, 2002.

[31] Sundaramoorthy, M., Terner J., \& Poulos, TL. Chemistry \& Biology. Vol. 5, 1998, 461-73.

[32] Zhao, Z., van Hooft, P., et al, Including the state of the art scientific workflow management systems in an e-Science environment. 1st IEEE e-Science 2005 conference, Melbourne Australia, 2005. 